J. Clin. Chem. Clin. Biochem.

Vol. 25, 1987, pp. $401-407$

(C) 1987 Walter de Gruyter \& Co.

Berlin - New York

\title{
Renal Ammonia Production from the Nitrogens of Glutamine in Intact Acidotic Dogs before and after Bicarbonate Infusions
}

\author{
By H. G. Preuss, Dianna Slemmer, J. Areas and J. Conrado \\ Department of Medicine (Nephrology), Georgetown University Medical Center, Washington, DC, USA
}

(Received December 2, 1986/April 15, 1987)

\begin{abstract}
Summary: Renal ammonia is produced from the amide nitrogen of glutamine, approximately $33-50 \%$. The remainder derives from the amino nitrogen of glutamine and other non amide sources, probably the amino nitrogens of other amino acids. We investigated the acute effects of acid-base perturbations on ammonia production from amide and non amide nitrogen sources to determine how they interrelate. Infusions of glutamine were given to some intact dogs to vary the renal load. Following an acute alkali challenge to dogs in metabolic acidosis, ammoniagenesis from the amide nitrogens decreased significantly when the presentation of glutamine to the kidney was normal or relatively low, but changed less or even increased when the glutamine load was relatively high. In contrast, ammonia from the non amide sources consistently decreased during acute alkalotic challenge at any glutamine load - high or low. Since decreased glutamine deamination leading to glutamate accumulation is generally associated with decreased deamidation in dogs with normal plasma glutamine concentrations, we explain the discrepancy of deamidation at high glutamine loads to an unmasking of a separate effect on the glutaminase (phosphate-dependent) pathway by the acute acid-base changes. Accordingly, our results indicate more than one influence from acute acid-base changes in vivo on renal ammonia formation, one stimulatory and other inhibitory. Nevertheless, the influence of glutamate removal predominates over the other effect on the phosphate-dependent glutaminase pathway at physiological concentrations of glutamine in the intact dog.
\end{abstract}

\section{Introduction}

Among the most investigated adaptations in renal biochemistry is the enbanced production of ammonia from glutamine during acidosis. It is generally accep= ted that the major augmentation of renal ammonia- genesis in acidotic dogs occurs through enhanced mitochondrial glutamine deamidation via phosphatedependent glutaminase and enhanced mitochondrial glutamate deamination via the glutamate dehydrogenase (GDH) pathways $(1-6)$.

Glutamine $\stackrel{\begin{array}{c}\mathrm{PO}_{4}^{3-} \\ \text { Phosphate-dependent }\end{array}}{\stackrel{\text { glutaminase }}{\longrightarrow} \text { Glutamate }} \stackrel{\substack{\mathrm{NAD}^{+} \\ \text {Glutamate }}}{\stackrel{\mathrm{NH}_{3}}{\text { (amide) }}}$ 2-Oxoglutarate


The precise stimuli which bring about the alterations in the glutaminase and glutamate dehydrogenase pathways are uncertain $(1,3,5)$. Importantly, the exact roles of acute perturbations in the environmental hydrogen ion concentration and/or bicarbonate ion concentration on renal ammonia metabolism in vivo are unclear.

Various in vitro studies which have concentrated on the effects of acute changes in hydrogen ion and/or bicarbonate concentrations on ammoniagenesis by renal tissues incubating in glutamine have reported disparate results. Using renal cortical tissue from dogs, one group found increased ammonia production when incubations at $\mathrm{pH} 7.0$ were compared to 7.7 (7). Others reported no changes $(8,9)$. In general, similar studies on rat slices showed no augmentation of ammoniagenesis $(10-14)$. In mitochondria incubating at lower medium $\mathrm{pH}$, ammoniagenesis from glutamine was relatively less even when rotenone was blocking the glutamate dehydrogenase pathway. Differently, lowering medium $\mathrm{pH}$ increases ammoniagenesis by mitochondria when 2-oxoglutarate is added to the medium (15). In isolated perfused kidneys of rats, ammoniagenesis increases or does not change in response to acute metabolic and respiratory acidosis $(16,17)$. What stimulation occurs is overcome when the urine is allowed to drain back into the reperfusate (18). We assume that many of the discrepancies found in in vitro studies are probably due to the different incubating conditions devised by the investigators. In contrast to the inability of acute increases in medium hydrogen ion concentration and decreases in bicarbonate concentration to consistently increase ammoniagenesis from glutamine in vitro, ammonia production regularly increases in slices and tubules under the same conditions as above when glutamate is the starting substrate $(19,20)$.

Utilizing information from previous in vitro studies (21), the purpose of the present investigation was to determine how acute changes in acid-base status of intact dogs influence the deamidation of glutamine as well as non amide ammonia formation. Since lactate metabolism, as well as glutamine metabolism, is affected during chronic acidosis (22), the renal uptake of this metabolite was also examined in some studies for comparative purposes.

\section{Methods}

Sixteen male and female chronically acidotic dogs with a weight range between 16 and $28 \mathrm{~kg}$ were used. We induced chronic metabolic acidosis in dogs by the daily administration of $10 \mathrm{~g}$ of ammonium chloride for 3-5 days. Dogs were anesthetized with i.v.-pentobarbital (approximately $20 \mathrm{mg} / \mathrm{kg}$ ) and addi- tional doses were given i.v. as needed. The femoral artery was cannulated for arterial sampling. Following an abdominal midline incision, both ureters were identified and catheterized close to the bladder. The right renal vein was entered via the femoral vein by manipulating a catheter with a curved tip into it by palpation. Dogs received 1 liter of normal saline to promote volume expansion.

The dogs were primed with $1.0 \mathrm{~g}$ creatinine, $0.2 \mathrm{~g}$ of $p$-aminohippurate and $0.88 \mathrm{~g}$ sodium sulfate dissolved in $20 \mathrm{ml}$ of water and given as an i.v. bolus. Throughout the study, the dogs were infused with a stock solution containing $5.0 \mathrm{~g}$ creatinine, $1.0 \mathrm{~g} \mathrm{p}$-aminohippurate, $3.26 \mathrm{~g}$ of sodium sulfate per liter of distilled water. In some dogs, a bolus of glutamine (1600 $\mu \mathrm{mol} \cdot \mathrm{kg}^{-1}$ ) was given followed by an infusion of the stock solution containing enough glutamine to deliver $40 \mu \mathrm{mol}$. $\mathrm{kg}^{-1} \cdot \min ^{-1}$. After a 30 min equilibration period, twó consecutive 20 min collection periods were begun (periods 1,2). After the initial control periods, an i.v. bolus containing $10 \mathrm{~g}-20 \mathrm{~g}$ of $\mathrm{NaHCO}_{3}$, was given over $10 \mathrm{~min}$ and the stock solution was changed to $6.3 \mathrm{~g} \mathrm{NaHCO}, 5.0 \mathrm{~g}$ creatinine and $1.0 \mathrm{~g}$ of $p$ aminohippurate per liter of water and contained enough glutamine to deliver $40 \mu \mathrm{mol} \cdot \mathrm{kg}^{-1} \cdot \mathrm{min}^{-1}$. Five $\mathrm{ml} \cdot \min { }^{-1}$ were given and equilibration was for at least one hour before two consecutive $20 \mathrm{~min}$ periods were run (periods 3,4 ).

Arterial and renal venous blood for ammonia determinations were drawn directly into lightly heparinized $20 \mathrm{ml}$ syringes containing $5.0 \mathrm{ml}$ of $100 \%$ carbon dioxide. The cold syringes were plunged into shaved ice after the blood was drawn. Blood ammonia determinations were performed within $30 \mathrm{~min}$ after drawing (23). Analyses were performed in duplicate or triplicate. A second sample of blood was obtained for the determination of blood p-amminohippurate, plasma creatinine and plasma substrates (24). Glutamine, lactate, alanine and glutamate were measured enzymatically as described in 1.c. (24). A third sample collected anaerobically was used to determine blood $\mathrm{pH}$ and $\mathrm{pCO}_{2}$ (Copenhagen Radiometer $\mathrm{pH}$ Meter with expanded scale) or plasma carbon dioxide content $\left(\mathrm{CO}_{2}\right.$ apparatus set method, Harleco Co., Gibbstown, New Jersey). The carbon dioxide content was used as an approximation of plasma $\left[\mathrm{HCO}_{3}^{-}\right]$in some studies.

Glomerular filtration rate (GFR) was measured by exogenous creatinine clearance. Renal blood flow (RBF), determined with $p$-aminohippurate, was measured by the Wolf modification of the Fick principle, that is, renal blood flow is calculated by V/U-Prv/Pa-Prv)

when

$\mathrm{V}$ is urine flow $\left(\mathrm{ml} \cdot \mathrm{min}^{-1}\right)$,

$\mathrm{U}$ is urinary concentration ( $\mathrm{mmol} \cdot \mathrm{ml}^{-1}$ ),

$\mathrm{Pa}$ is plasma arterial concentration $\left(\mathrm{mmol} \cdot \mathrm{ml}^{-1}\right)$ and

Prv is plasma renal venous concentration $\left(\mathrm{mmol} \cdot \mathrm{ml}^{-1}\right)$.

Total renal ammonia production was calculated from the urinary excretion plus the amount added to the renal venous blood. The addition or extraction of amino acids and lactate by the kidneys was calculated and expressed as $\mu \mathrm{mol} \cdot \mathrm{min}^{-1}$. Corrections were made for renal venous concentrations to account for water lost in urine formation (25). Renal plasma flow equalled

[RBF $\times(1-\mathrm{PCV})]$,

where

PCV is packed cell volume, haematocrit.

Appropriate statistical analyses were performed with either paired or group analysis by using Student's " $t$ " test. Regression lines were computed by the least squares method using a Sharp Compet CS 4500 calculator. An "F" test was used to compare whether the slopes of the linear regression lines were statistically different.

The following estimates were made: 
Total ammonia produced

The sum of the ammonia excreted per minute plus the difference between that leaving the renal vein and that arriving in the arterial blood is the total ammonia produced (24).

\section{Amide ammonia (glutamine deamidation)}

Very little glutamine is excreted even when infused at high concentrations (26). Therefore, amide nitrogen deamidation is the difference between the amount presented to the kidney in the arterial blood and that leaving in the renal vein. This is a theroretical maximal amount of amide nitrogen removed for ammonia production, but a good estimate since Stone \& Pitts have shown that very little renal ammonia appears again as amino or amide nitrogen (27). Comparison of in vivo studies in which ${ }^{15} \mathrm{~N}(26)$ and this methodology (24) were used indicate the suitability of the estimate. Accordingly, the terms "glutamine deamidation" and "amide ammonia" formation are used interchangeably throughout the text.

\section{Non-amide ammonia}

This is a theoretical maximal amount of ammonia formed from amino nitrogen and other non amide sources and is derived by subtracting amide ammonia formed from the total ammonia production (24). While the estimate may include ammonia formed from non amino sources, we believe this to be small (28). The use of exogenous glutamine in some of the studies should proportionately lessen the importance of a non amino source (26).

\section{Glutamine load}

This is calculated as the renal plasma flow times the arterial glutamine concentration.

\section{Glutamate and alanine release and lactate uptake}

This is the difference between the arterial and renal venous concentrations corrected for urine extraction (25) times the renal plasma flow.

\section{Results}

Data from 16 dogs are depicted in table 1. Dogs ingesting $\mathrm{NH}_{4} \mathrm{Cl}$ for at least 3 days were examined during the first 2 periods (baseline) and then challenged with $\mathrm{NaHCO}_{3}$ infusions for 2 periods (test) to bring about acute alterations in acid-base status. Results from the baseline and test periods were averaged. Circulating hydrogen ion and bicarbonate concentrations decreased and increased respectively from baseline in. every experiment following bicarbonate infusions.

Five dogs in Group 1 received only bicarbonate infusions during test periods, whereas 7 dogs in Group 2 and 4 dogs in Group 3 received glutamine infusions throughout all periods in addition to bicarbonate during the test periods. In Groups 1 and 2, the $\mathrm{pCO}_{2}$ remained relatively constant between the baseline and test periods whether the initial value was relatively

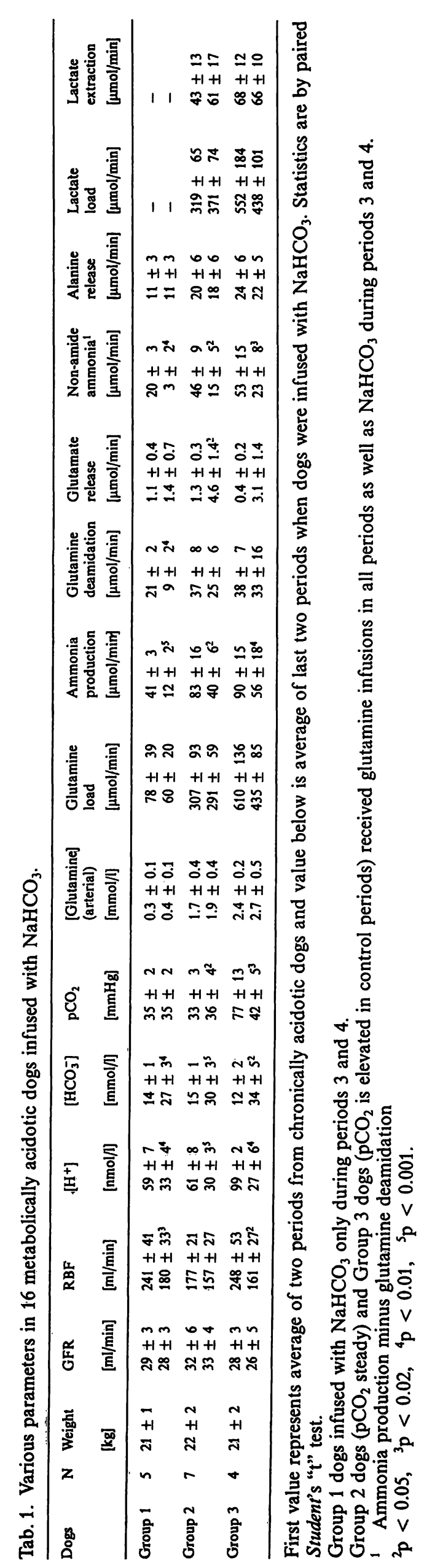


normal, low or high. Dogs in Group 3 had a large decrease in $\mathrm{pCO}_{2}$ after bicarbonate infusions from an elevated $\mathrm{pCO}_{2}$ in the baseline periods. Accordingly, these dogs were undergoing respiratory as well as metabolic changes in their acid-base status. This caused a more severe change in hydrogen ion concentration.

In Group 1 dogs, infusions of $\mathrm{NaHCO}_{3}$ brought about many expected effects - lowered hydrogen ion concentration, ammonia production, glutamine deamidation (amide ammonia formation) and nonamide ammonia formation. GFR, $\mathrm{pCO}_{2}$, arterial glutamine, glutamine loads, glutamate and alanine release were not changed significantly. Although average glomerular filtration rate remained near constant, renal blood flow decreased. This disparity between filtration rate and blood flow generally held in all the groups studied.

The infusion of glutamine magnified many of the investigated metabolic parameters (Group 2) and caused some differences from the observations noted in Group 1. Obviously the average arterial glutamine concentrations and renal glutamine loads were higher. An enhanced glutamate release during $\mathrm{NaHCO}_{3}$ infusions became apparent under these conditions. Although total ammonia production and non-amide ammonia formation still decreased markedly during alkali infusion, the difference in glutamine deamidation was lessened and missed statistical significance in the 2 groups. Even though the changes in acidbase balance were more severe in the dogs in Group 3 due to the changes in $\mathrm{pCO}_{2}$, the results were very similar to those found in Group 2. In neither of these last 2 groups was alanine release, lactate loads or renal lactate extraction influenced to any great extent by bicarbonate infusions.

As a first approximation, we made the correlations using data from dogs in Groups 1,2 and 3. Some of these are depicted in figures 1 and 2 . Significant positive correlations were found between glutamine loading and a number of parameters, i. e., ammonia production, glutamine deamidation (amide ammonia formation) and non-amide ammonia formation in both the values derived from the baseline periods and the periods during bicarbonate infusion.

We compared the correlations between the baseline and test periods in the 16 dogs for total ammonia formation at different glutamine loads. A steady increase in ammonia formation was seen with increasing glutamine loading. The slopes during chronic acidosis $(\mathrm{y}=0.11 \mathrm{x}+38.0, \mathrm{r}=0.84, \mathrm{p}<0.01)$ and after bicarbonate infusions $\left(y=0.12 x+5.0^{\circ}\right.$,

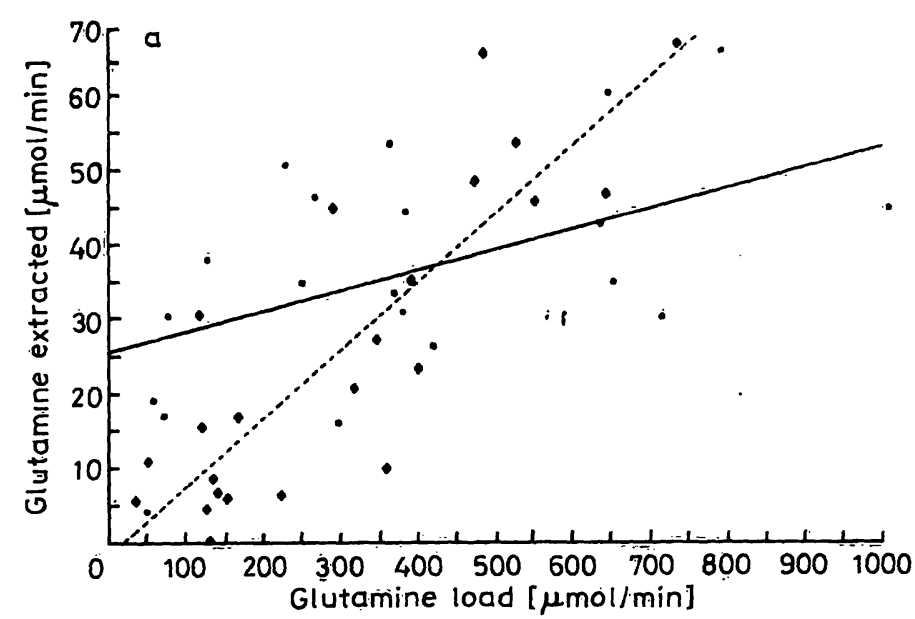

Fig. 1 a. Correlation between renal glutamine load and glutamine extracted in chronically acidotic dogs (solid line) and after they were made acutely alkalotic (dotted line).

Acidosis $\circ(\mathrm{r}=0.65, \mathrm{p}<0.01, \mathrm{~m}=0.027)$.

Alkalosis $(r=0.85, \mathrm{p}<0.01, \mathrm{~m}=0.090)$

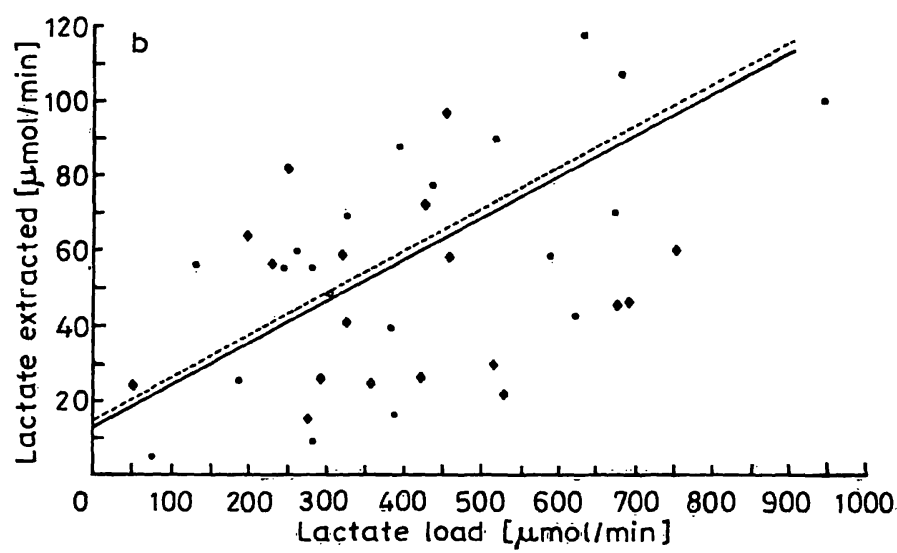

Fig. 1 b. Correlation between lactate load and lactäte extraction in chronically acidotic dogs (solid line) and after they were made acutely alkalotic (dotted line)

Acidosis $\bullet\left(r=0.80, \mathrm{p}_{\mathrm{r}}<0.01, \mathrm{~m}=0.11\right)$

Alkalosis $\diamond(\mathrm{r}=0.59, \mathrm{p}<0.01, \mathrm{~m}=0.11)$

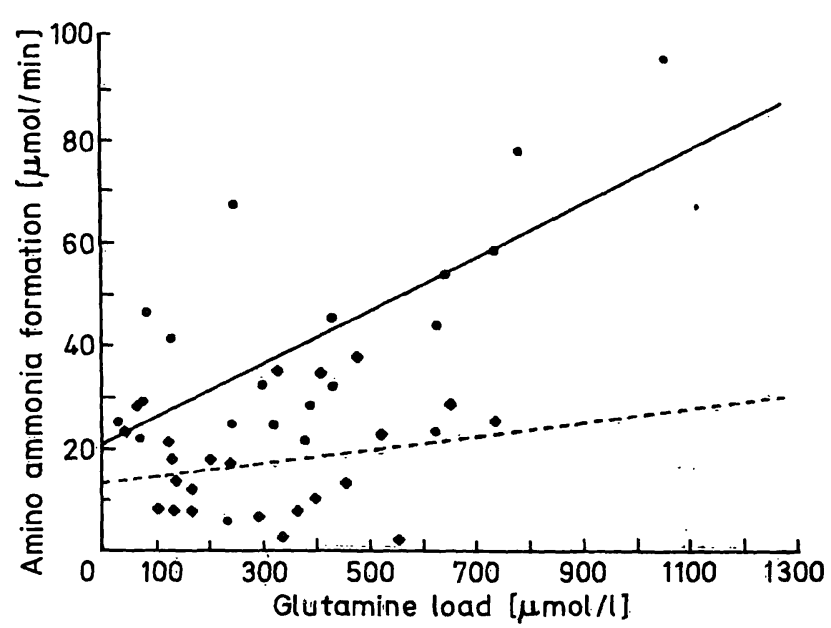

Fig. 2. Correlation between renal glutamine load and estimated amino ammonia formation (total ammonia formation minus glutamine extrāction) in chronically acidotic dogs (solid line) and after they were made acutely alkalotic (dottes lines).

Acidosis $\bullet(r=0.64, p<0.01, m=0.05)$

$y=0.05 x+20.6$

Alkalosis $(\mathrm{r}=0.10, \mathrm{p}=\mathrm{NS}, \mathrm{m}=0.01)$

$y=0.01 x+13.9)$ 
$r=0.85, p<0.01)$ were essentially parallel, although values following infusion ran below the acidotic ones.

Glutamine extraction, i.e., deamidation, per given load was greater in acidosis at the lower glutamine loads; however, it was less when glutamine perfusing the kidneys exceeded $400 \mu \mathrm{mol} \cdot \mathrm{min}^{-1}$ (fig. $1 \mathrm{a}$ ). Indeed, the 2 lines crossed; because the slope of correlation was steeper during alkalosis $(p<0.01)$. There was also a significant positive correlation between the lactate extracted by the kidney and the lactate load under both acid-base conditions (fig. 1 b). However, unlike glutamine deamidation the lines of correlation were parallel during chronic acidosis and after acute bicarbonate loading. In contrast to amide ammonia formation via glutamine deamidation, ammonia formation from non amide sources (total ammonia formation minus glutamine extraction) showed both a steeper slope $(p<0.05)$ and higher values in the acidotic periods (fig. 2). When comparing glutamate release during glutamine loading, there was a significant positive correlation during bicarbonate infusion, $(y=0.008 x+1.0, r=0.57$, $\mathrm{p}<0.05$ ) while the line was flat during acidosis ( $y$ $=0.001 \mathrm{x}+1.0, \mathrm{r}=0.08$, not significant).

\section{Discussion}

A paradox exists in the association between acidosis and augmented renal ammoniagenesis. Blood hydrogen ion concentrations increase and bicarbonate ion concentrations decrease to a maximum within hours after initiation of acute acidosis. These acute changes in acid-base parameters usually represent the greatest extremes, because both hydrogen ion and bicarbonate concentrations gradually decrease and increase respectively toward baseline despite persistent acid challenge (2). Nevertheless, adaptive ammoniagenesis continues to increase stepwise to a maximum over days (3) even though hydrogen ion and bicarbonate concentrations are approaching normality.

In vitro studies suggest that glutamine metabolism changes in at least two respects in response to acidbase perturbations. The in vitro studies with glutamate as the ammonia precursor suggest that the glutamate dehydrogenase pathway is stimulated by the acidic environment, whereas, those with glutamine as the ammonia precursor suggest that an "acidic medium" inhibits the glutaminase pathway even though the more rapid removal of the glutamate (glutamate dehydrogenase pathway) formed from glutamine should augment deamidation (21). Two separate effects could explain the inconsistencies in in vitro production previously noted $(9,11-13,15)$. Enhanced deamination via greater glutamate removal through the glutamate dehydrogenase pathway and depressed deamidation of the glutamine molecule under acidic conditions occurred simultaneously and mitigated the divergent effects of one another on ammoniagenesis (21). Is the same true in vivo?

In the present in vivo study, we chose to follow the conversion of metabolic acidosis to the normal state or alkalosis by means of $\mathrm{NaHCO}_{3}$ infusions to assess acute perturbations in acid-base balance in vivo, because we found in comparison that acute acid challenge of alkalotic or normal dogs decreased glomerular filtration rate and renal blood flow far more. Marked changes in filtration rate and blood flow in response to acute augmentation of hydrogen ion concentration have been noted previously $(30-32)$.

Some dogs were infused with glutamine to magnify precursor metabolism. By this means, we had previously been able to enhance our capability to estimate the amide and non amide nitrogens' contributions to ammoniagenesis in intact dogs (24). Infusions of glutamine have long been known to enhance ammoniagenesis $(25,26)$ and magnify glutamine metabolism in the kidney $(24,26)$. This increase is in amide and non-amide ammonia formation (26). We approximated amide and non-amide ammonia formation by measuring total ammonia formation and glutamine deamidation. Using this methodology, previous in vitro (20) and in vivo studies (24) correlated quite closely with results obtained using ${ }^{15} \mathrm{~N}$ labels on precursors $(4,33)$.

The results found in the 5 dogs receiving no glutamine loading (Group 1) were not unexpected. Bicarbonate infusions lowered overall ammonia production, and this was due both to decreased amide and non-amide ammonia formation. No significant changes were detected in glutamate or alanine release under baseline and test conditions. However, some differences were noted in the experiments with glutamine loading. Although the $\mathrm{pCO}_{2}$ was fairly constant indicating mainly metabolic alterations between the baseline and test periods in the Group 2 dogs and decreased from high level in Group 3 indicating concurrent metabolic and respiratory alterations, the results were similar and will be discussed together. The changes produced by bicarbonate infusions in total ammonia formation and non-amide ammonia corroborated those found without glutamine loading, but amide ammonia formation was no longer significantly decreased, and glutamate release rose significantly (compare Groups 2 and 3 with Group 1). The latter 
indicated that the changing acid-base status was influencing the glutaminase and glutamate dehydrogenase pathways differently simulating in vitro findings (21).

Further support was obtained by examining the proportion of total ammonia derived from amide and non-amide nitrogens. These increased and decreased relatively during the bicarbonate challenge. During chronic acidosis, amide ammonia formation was $51 \%$ of the total ammonia formed in Group 1; and during glutamine loading, amide ammonia formation was $45 \%$ to $42 \%$ of the total in Groups 2 and 3 . The magnitude of these results agree with the percent of ammonia formed from the amide nitrogen of glutamine in ${ }^{15} \mathrm{~N}$ studies during acidosis reported by Pitts \& Pilkington (26). In the alkalotic period, the percent of amide ammonia to total ammonia formation rose to $75 \%, 64 \%$ and $59 \%$ respectively in the three groups.

Unfortunately, there is no ${ }^{15} \mathrm{~N}$ data in alkalosis to compare these percents. Because of this, a relative increase in renal glutamine content during alkalosis could explain the high apparent rate of deamidation after bicarbonate infusions. This is unlikely for 3 reasons. Previous investigations have shown no differences in luminal transport of glutamine at high loads during acidosis and alkalosis (34). In addition, renal alanine concentrations rise with glutamine loading, but are not different during acidosis and alkalosis suggesting no difference in glutamine uptake. Finally, renal glutamine content was measured in 4 dogs receiving glutamine infusions to increase plasma levels 3 times above normal $\left[1.8 \mu \mathrm{mol} \cdot \mathrm{ml}^{-1} \pm 0.5\right.$ (SEM) in acidosis and $1.7 \mu \mathrm{mol} \cdot \mathrm{ml}^{-1} \pm 0.2$ (SEM) in alkalosis]. Renal glutamine concentration was higher during acidosis $\left[12.9 \mu \mathrm{mol} \cdot \mathrm{g}^{-1} \pm 4.8\right.$ (SEM) compared to $\left.8.5 \mu \mathrm{mol} \cdot \mathrm{g}^{-1} \pm 3.6(\mathrm{SEM}), \mathrm{p}<0.05\right]$. The $57 \%$ increase in renal glutamine content in acidosis correlates closely with the $47 \%$ higher glutamine load during acidosis, $290 \mu \mathrm{mol} \cdot \mathrm{min}^{-1} \pm 32$ (SEM) versus $197 \mu \mathrm{mol} \cdot \mathrm{min}^{-1} \pm 7$ (SEM). Based on the above, we agree with Pitts' previous assumption that there is probably no change in renal glutamine uptake arising from acid-base perturbations.

Figure 1 depicts our attempt to discern more clearly the differences in glutamine deamidation of the 3 groups of dogs brought on by the acute alkali challenge. When glutamine deamidation was plotted against glutamine load, significantly positive correlations were found for the periods of chronic metabolic acidosis and the test periods when hydrogen ion concentration was near or below normal. The lines of correlation for both the chronic metabolic acidotic periods and the periods during bicarbonate infusion crossed. At lower loads, glutamine deamidation per given glutamine load was greater in acidosis, but at higher loads was greater after bicarbonate infusion (fig. $1 \mathrm{a}$ ). This differed from non-amide ammonia production which was less during alkali challenge at lower glutamine loads and remained less as the glutamine load increased (fig. 2).

The obvious rise in glutamate release during bicarbonate infusion was consistent with the lower nonamide ammonia formation. It is generally felt that the non-amide ammonia formation derives from amino nitrogen (28). Other than glycine, most amino acids contribute to ammonia production by transamination of 2-oxoglutarate to form glutamate and by subsequent oxidative deamination of glutamate to liberate ammonia (35). It seems likely that similar to the in vitro condition (21) the opposing effects of bicarbonate infusions on amide and amino ammonia formation cancel each other, at least to some extent, and are responsible for the near similar slopes correlating total ammonia formation with glutamine loading.

It is generally accepted that the glutamate dehydrogenase pathway through glutamate removal is the major regulator of the glutaminase pathway at physiological glutamine loads $(24,36)$. We were not surprised that less non-amide and amide ammonia were formed following bicarbonate infusions at the lower glutamine loads. However, higher amide ammonia formation (with higher glutamine loads) during alkalotic challenge suggests that a factor influencing glutaminase pathway activity other than glutamate removal through the glutamate dehydrogenase pathway became rate limiting. In further support, almost no glutamate dehydrogenase activity is present in the case of alkalosis (24). Therefore, control of the glutaminase pathway cannot be ascribed to the glutamate dehydrogenase pathway under this condition.

Based on in vitro studies, the best possibility to explain the above is that lessened deamidation during acidosis may relate to the hydrogen ion and/or bicarbonate concentrations affecting the activity of the glutaminase enzyme in mitochondria. Glutaminase is more active at alkalotic range hydrogen ion concentrations in dog and rat kidneys $(21,29,36)$. Accordingly, the hydrogen ion concentration may have a greater influence on deamidation (glutaminase activity) than glutamate removal (37) at high loads. Although we cannot prove it conclusively, the increased hydrogen ion concentration during acute acidosis should also influence glutaminase activity at low glutamine loads, i. e., normal plasma concentrations. If so, the more rapid removal of glutamate during acidosis $(21,24)$ has greater influence on the glutaminase 
pathway activity than the hydrogen ion effect on glutaminase at the physiological concentrations of circulating glutamine.

Another possibility to explain altered glutaminase activity by hydrogen ion concentration is an effect on mitochondrial transport. Against this, the decreasing hydrogen ion concentration during bicarbonate infusions should decrease active transport of glutamine (38) and lower deamidation markedly, just the opposite of what happened.

The magnitude of the renal lactate load does not alter lactate extraction in response to acid-base changes (fig. $1 \mathrm{~b}$ ). This does not mean that renal lactate metabolism has not been influenced in contrast to glutamine. Pitts (22) found that ${ }^{14} \mathrm{CO}_{2}$ production from

\section{References}

1. Van Slyke, D. D., Phillips, R. A., Hamilton, P. B., Archibald, R. M., Futcher, P. H. \& Miller, A. (1943) J. Biol. Chem. 150, 481-482.

2. Owen, E. E. \& Robinson, R. R. (1963) J. Clin. Invest. 239, 263-276.

3. Pitts, R. F. (1973) In: Handbook of Physiology, Section 8, Am. Physiol. Soc., pp. 455-496.

4. Pitts, R. F., Pilkington, L. A. \& DeHaas, J. C. M. (1965) J. Clin. Invest. 44, $731-745$.

5. Tannen, R. L. (1978) Am. J. Physiol. 235, F265-277.

6. Halperin, M. L., Goldstein, M. B., Stinebaugh, B. J. \& Junges, R. L. (1985) In: The Kidney: Physiology and Pathophysiology (Seldin, D. W. \& Giebisch, G., eds.) Raven Press, New York, N. Y. pp. 1471-1490.

7. Pilkington, L. A. \& O'Donovan, D. J. (1971) Am. J. Physiol. 220, 1634-1639.

8. Cartier, P., Belanger, P. \& Lemieux, G. (1975) Am. J. Physiol. 222, 934-943.

9. Vinay, P., Lemieux, G., Gougoux, A. \& Lemieux, C. (1980) Int. J. Biochem. 12, 89-98.

10. Alleyne, G. A. O. (1970) J. Clin. Invest. 40, 943-951.

11. Goodman, A. D. (1973) J. Láb. Clin. Med. 81, 905-918.

12. Irias, J. J. \& Greenberg, R. E. (1972) Am. J. Physiol. 223, 1128-1134.

13. Pagliara, A. S. \& Goodman, A. D. (1970) J. Clin. Invest. 49, $1967-1974$.

14. Relman, A. S. \& Narins, R. G. (1975) Med. Clin. N. Am. $59,583-593$.

15. Simpson, D. P. (1980) J. Biol. Chem. 255, 7123-7128.

16. Hems, D. A. (1972) Biochem. J. 130, 671-680.

17. Tannen, R. L. \& Ross, B. D. (1979) Clin. Sci. 56, 353- 364.

18. Terao, N. \& Tannen, $\vec{R}$. L. (1980) Am. J. Physiol. 239, F445-451.

19. Preuss, H. G. (1969) Nephron 6, 235-246.

20. Preuss, H. G. (1971) Am. J. Physiol. 22, 54-58. lactate decreases during chronic acidosis. The discrepancy could happen if decreased oxidation was balanced by an increased gluconeogenesis with less $\mathrm{CO}_{2}$ production (39). This would explain an unchanged extraction in the face of changing ${ }^{14} \mathrm{CO}_{2}$ production. Alternatively, ${ }^{14} \mathrm{CO}_{2}$ production might not change during acute acidosis like in chronic acidosis. Obviously, this point will need further clarification.

\section{Acknowledgement}

The authors are grateful for the excellent secretarial assistance of Mrs. Betty Mendelson and Mrs. Elizabeth Lightfoot-Ergueta and the assistance with the statistics of Dr. Susan Ahmed.

Supported by NIH Grant AM 27633 and a grant from the American Heart Association, Nations Capital Affiliate.

21. Preuss, H. G., Vavatsi-Manos, O., Vertuno, L. L. \& Baird, K. (1974) Proc. Soc. Exper. Biol. Med. 146, 803-808.

22. Pitts, R. F. (1975) Med. Clin. N. Am. 59, 507-518.

23. Preuss, H. G., Bise, B. W. \& Schreiner, G. E. (1966) Clin. Chem. 12, 329-377.

24. Lombardo, J. V., Risquez, A., McCarthy, M. \& Preuss, H. G. (1981) Kidney Int. 49, 540-552.

25. Denis, G., Preuss, H. \& Pitts, R. F. (1964) J. Clin. Invest. 43, $571-582$.

26. Pitts, R. F. \& Pilkington, L. S. (1966) J. Clin. Invest. 45, 86-93.

27. Stone, J. W., Balagura, S. \& Pitts, R. F. (1967) J. Clin. Invest. 46, 1603-1608.

28. Pitts, R. F., DeHaas, J. \& Klein, J. (1963) Am. J. Physiol. 204, 187-191.

29. Sayre, E. W. \& Roberts, E. (1958) J. Biol. Chem. 233, $1128-1134$.

30. Fine, A., Bennett, F. I. \& Alleyne, G. A. O. (1978) Clin. Sci. Mol. Med. 54, 503-508.

31. Gougoux, A., Vinay, P., Cardosa, M., Duplain, M. \& Lemieux, G. (1982) Am. J. Physiol. 243, F227-F234.

32. Weiss, F. R. \& Preuss, H. G. (1971) Nephron 8, 344-354.

33. Nissim, I., Yudkoff, M. \& Segal, S. (1985) J. Biol. Chem. 150, 13955-13967.

34. Pilkington, L. A., Young, T. K. \& Pitts, R. F. (1970) Nephron 7, 51-60.

35. Braunstein, A. E. \& Kritzman, M. G. (1937) Enzymologia $2,129-137$

36. Pollack, V. E., Mattenheimer, H., DeBruin, H. \& Weinman, K. J. (1965) J. Clin. Invest. 44, 169-181.

37. Preuss, H. G., Vavatsi-Manos, O., Eastman, S. T. \& Gaydos, D. (1981) Nephron 27, 244-253.

38. Simpson, D. P. \& Adams, W. (1975) J. Biol. Chem. 250, $8148-8158$

39. Krebs, H. A. (1975) Med. Clin. N. Am. 59, 519-522.
Harry G. Preuss, MD

Professor of Medicine \& Pathology

Georgetown University Medical Center

153 Basic Science Building

4000 Reservoir Rd., NW

Washington, DC 20007

USA 


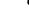

